\title{
Optimization of Sorting Conditions for the Selection of Stable, High-Producing Mammalian Cell Lines
}

\author{
Max Zeyda, Nicole Borth,* Renate Kunert, and Hermann Katinger \\ Institute for Applied Microbiology, University of Agriculture, Muthgasse 18, 1190 Vienna, Austria
}

\begin{abstract}
The production of Green Fluorescent Protein in recombinant NIH3T3 mouse fibroblast cells was used as a model to determine the optimal conditions for the rapid isolation of high-producing cell lines with a fluorescence-activated cell sorter. "Bulk sorting", that is, sorting of a large number of positive cells, did not result in a stable, highproducing cell line due to overgrowth of high-producing cells by low- or nonproducing cells. The production kinetics and expression of GFP during batch culture was found to differ between NIH3T3 cells and HepG2 hepatoma cells, even though the same plasmid was used for transfection. The kinetics of product formation need therefore to be determined from case to case to select the optimal timepoint for analysis and sorting. Subcloning of sorted cells into mi crotiter plates only resulted in high-producing subclones when 1 or 2 cells were seeded per well. Higher seeding rates again resulted in overgrowth of low- or nonproducers. By subcloning, two high-producing cells lines could be isolated. They had a 10- and 15-fold higher fluorescent signal compared to the negative control. While one of these subclones started to decrease it's GFP expression after 2 months, the other clone stably expressed GFP for 4 months.
\end{abstract}

\section{Introduction}

The economic production of recombinant proteins in mammalian cells is highly dependent upon the selection of high-producing cell lines. This process is extremely time-, labor-, and cost-consuming. Its success highly depends on the skill and experience of the researcher undertaking this process. Due to the high cost and the duration of screening, the use of a flow cytometer and cell sorter for the enrichment of high-producing cell lines is an attractive alternative. Flow cytometry can measure the fluorescent signal of single cells and separate cells with the desired properties from a mixed population. This would allow us to concentrate the work of cloning, screening, and testing on cells with an increased likelihood of high production rates. Cells as rare as $10^{-6}$ can be isolated by this methodology in a reasonable time frame using modern high-speed sorters (Rosenblatt et al., 1997). Some examples of the application of this methodol ogy are the sorting of hybridoma cells according to their surface IgG expression, which in many cases was found to correl ate to the specific secretion rates (Marder et al., 1990; Sen et al., 1990; McKinney et al., 1991; Kromenaker and Srienc, 1994; Cherlet et al., 1995); the selection of cell lines with other surface-expressed proteins (Morrison et al., 1997); and the use of $\beta$-galactosidase and, more recently, of GFP for the optimization of transient and stable gene expression (see Lybarger et al., 1996; Subramanian and Srienc, 1996; Zhang et al., 1996; and Gubin et al., 1997 as some examples). The main problem encountered in the selection of recombinant high-producing cell lines is the instability of product expression even in the presence of a selective marker (Gu et al., 1992; Kim et al., 1998; Lybarger et al., 1996). This instability together with a frequently observed reduction

* Corresponding Author. Tel: +43/1/36006-6232. Fax: +43/1/ 369 7615. E-mail: borth@mail.boku.ac.at. of growth rates at increased production rates will constantly reduce the number of producing cells in a population: a growth advantage of as little as $10 \%$ will be sufficient (Kromenaker and Srienc, 1994).

In the present study we wanted to determine which basic strategies have to be considered when high-producing subclones are to be selected from a mixed population of non-, low, and high producers. In principle there are two options. One is to sort a large number of positive cells into a single culture vessel ("bulk sorting"). Theresulting populations will be mixed, consisting of cells with various specific production rates. The advantage of this strategy is that one can quickly obtain a relatively large population of cells, which is why this method is used in all cited papers about cell sorting. The disadvantage, however, is that the resulting population will be polyclonal with the danger of instability and overgrowth of low producers.

To avoid this problem, one can clone sorted cells directly into microtiter plates. By this strategy it will take longer to obtain a working stock of sufficient cells. They will be clonal and more homogeneous, however. In the present study we have tried to determine which strategy will most likely result in the selection of a stable, highproducing cell line. Additional factors, such as the kinetics of product formation, were also considered.

\section{Material and Methods}

Cell Lines and Media. NIH3T3 mouse fibroblast cells (ATCC CRL 1558) were passaged 1:10 every 3-4 days in DMEM/Ham's F 12 1:1, containing 4 mM glutamine and $10 \%$ foetal calf serum (FCS). HepG2 liver carcinoma cells (ATCC HB 8065) were passaged 1:10 once a week in DMEM/Ham's F 12 1:1 containing 4 mM glutamine and $10 \%$ FCS. Cells were incubated in a humid chamber containing $7 \% \mathrm{CO}_{2}$ in air at $37^{\circ} \mathrm{C}$. Media were from Seromed (F RG), FCS was from Gibco Life Technologies (UK), culture vessels were from Nunc (Denmark), and all other reagents were from Sigma (USA). 

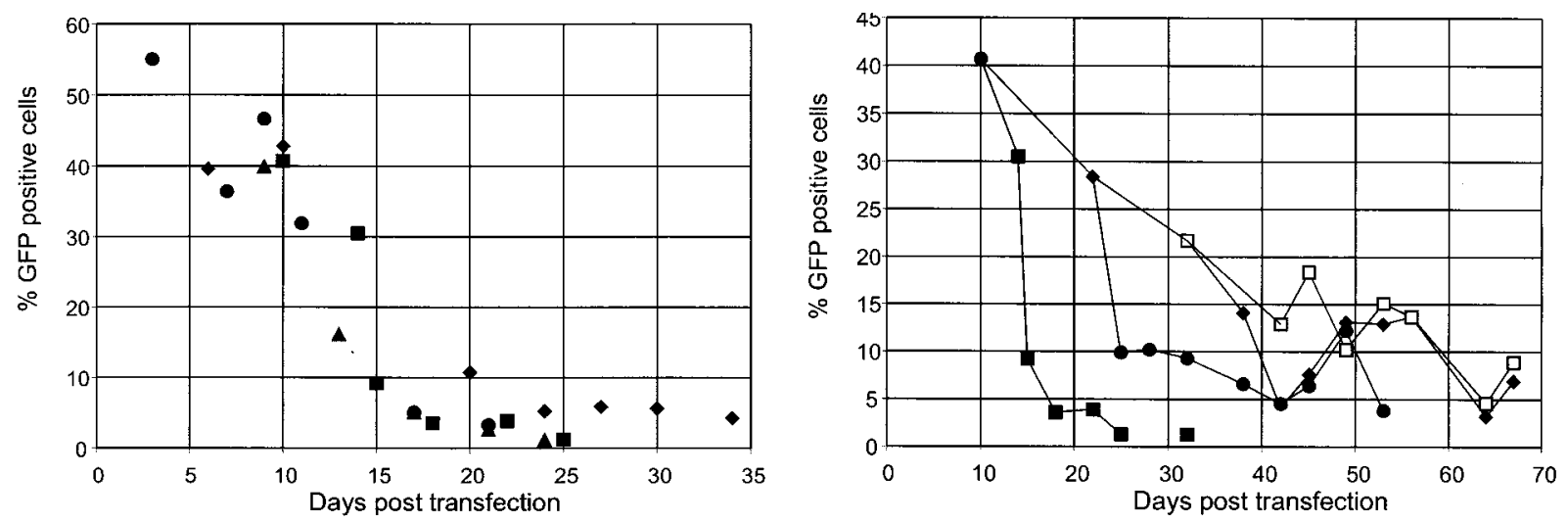

Figure 1. Left: Percentage of GFP positive NIH3T3 cells. Four transfections at different times were analyzed. Right: Percentage of GFP positive cells after bulk sorting. Cells were sorted 10 days after the transfection ( $\boldsymbol{\square}$ transfection, $\bullet$ first sort). This was repeated twice ( second sort, $\square$ third sort).

Plasmid and Transfection. Cells were passaged 1:2 $24 \mathrm{~h}$ before transfection. For one $75 \mathrm{~cm}^{2}$ T-flask $0.5 \mathrm{~mL}$ $0.25 \mathrm{M} \mathrm{CaCl}_{2}$ solution containing $10 \mu \mathrm{g}$ of plasmid, pGFP-C1 (Clontech, Palo Alto, CA) were added dropwise to $0.5 \mathrm{~mL} 2 \times$ Hanks Balanced Salt Solution. After $1 \mathrm{~h}$ the culture supernatant of the $T$ flask was reduced to 10 $\mathrm{mL}$ and the crystal suspension was added. After $4 \mathrm{~h}$ of incubation at $37^{\circ} \mathrm{C}$ cells were shocked with $5 \mathrm{~mL}$ of $10 \%$ DMSO in medium. After 7 min the DMSO solution was removed, cells were washed $3 \times$ with medium and incubated overnight, before selective pressure $(0.5 \mathrm{mg} / \mathrm{mL}$ G418) was added. When cells started to divide (usually after 5-10 days), they were passaged at the required dilution rates.

Flow Cytometric Analyses and Cell Sorting. Cells were suspended by trypsinisation and washed with medium containing FCS. After centrifugation, cells were resuspended in PBS and measured on a FACS Vantage equipped with a $6 \mathrm{~W}$ argon Laser (Coherent) tuned to $488 \mathrm{~nm}$ at $100 \mathrm{~mW}$ output power. GFP fluorescence was measured through a 530/30BP filter. For analysis a marker was selected of nontransfected control cells that contains less than $2 \%$ cells with the highest GFP fluorescence. Transfected cells were then analyzed using the same marker and the percentage of cells with high fluorescence determined (= "\% positive cells"). F or sorting, live cells were selected according to their FSC/SSC histograms and plotted into a dot plot of FSC-width (to excl ude cell doublets) versus FII (GFP fluorescence). For the subcloning experiments, cells were sorted directly into 96-well plates using the ACDU Automatic Cell Deposition Unit.

Surface Staining for Determination of Cell Growth. Cells were washed with PBS, carefully resuspended in $50 \mu \mathrm{L}$ of Diluent $\mathrm{C}$ from the Zynaxis PKH26GL Kit (Sigma), and mixed with $10 \mu \mathrm{M}$ dye. After $3 \mathrm{~min}$ $100 \mu \mathrm{L}$ of FCS was added, and after another minute, 1 $\mathrm{mL}$ of PBS was added. After centrifugation, cells were resuspended in $100 \mu \mathrm{L}$ of $20 \%$ FCS in PBS, transferred into a fresh tube, and diluted with $1 \mathrm{~mL}$ of $20 \%$ FCS in PBS. Cells were resuspended in medium. GFP fluorescence was measured on FI-1 through a 530/30 BP filter, PK H26 fluorescence on FI-2 through a 575/26 BP filter. Unstained GFP producing cells and nontransfected PKH26 stained cells were used for compensation setup.

\section{Experimental Procedure and Results}

Transfection and Bulk Sorting. NIH 3 T 3 cells were transfected 4 times with plasmid pGFP-C1. The percentages of GFP positive cells are presented in Figure 1.
The number of GFP positive cells decreased to less than $6 \%$ after 2-3 weeks. To enrich for high producers, we sorted the $10 \%$ cells with the highest fluorescence from Transfection ( $\mathbf{\square}$ in Figure 1) 10 days after transfection. Approximately 5000 cells were sorted into a $2 \mathrm{~cm}^{2}$ well. Confluent cells were transferred into a $10 \mathrm{~cm}^{2}$ well. When confluence was reached (usually after 10-12 days), cells were analyzed and sorted again. In the second round of sorting the $1 \%$ best GFP producers were selected and in the third sort the $4 \%$ best GFP producers. In all cases the decrease in positive cells was delayed compared to the starter population, but the percentage of positive cells as well as the geometric mean of the whole population decreased steadily until a stable population containing $5-15 \%$ positive cells was reached (Figure 1 ). The reduction in the percentage of positive cells sorted in the second and third sort did not help in establishing a stable high producer. However, in the unsorted population, the fluorescent signal had decreased to the value of the negative control 20-30 days after transfection.

Batch Cultures and Growth Dependence of GFP Production. I nitial transfection experiments revealed a very high variation in the fluorescence signals between consecutive measurements of comparable populations. To reveal the reason for this variation, we ran a batch culture of bulk sorted NIH3T3 cells. Cells were diluted 1:5 and 1:7 and measured daily (Figure 2). The concentration of GFP in growing cells is very low and increases after cells reach confluence (cells diluted 1:5 were confluent after $2-3$ days, those diluted 1:7 after 4 days). The fluorescent signal is lower, the lower the cell density. There are two possible explanations for this behavior: one is that the promoter controlling transcription of the GFP gene is most active when cells are quiescent. This is very unlikely as the GFP gene is under the control of the CMV promoter, a strong viral promoter. Such promoters are either constitutively expressed or are preferentially active during S-phase at high growth rates (Gu et al., 1993). The other possibility is that although GFP is constitutively expressed, the simultaneous production of biomass during high growth rates will dilute the product, so that the low concentrations are undetectable (Subramanian and Srienc, 1996). Only after biomass production stops can the relative GFP concentration increase. This behavior, however, seems to be cell linespecific. We have al so determined the fluorescence signal from a HepG2 cell line transfected with the same plasmid. These cells were passaged 1:10 and analyzed for 10 days (Figure 2). After 3 and 7 days the culture supernatant was replaced by fresh medium. In contrast 

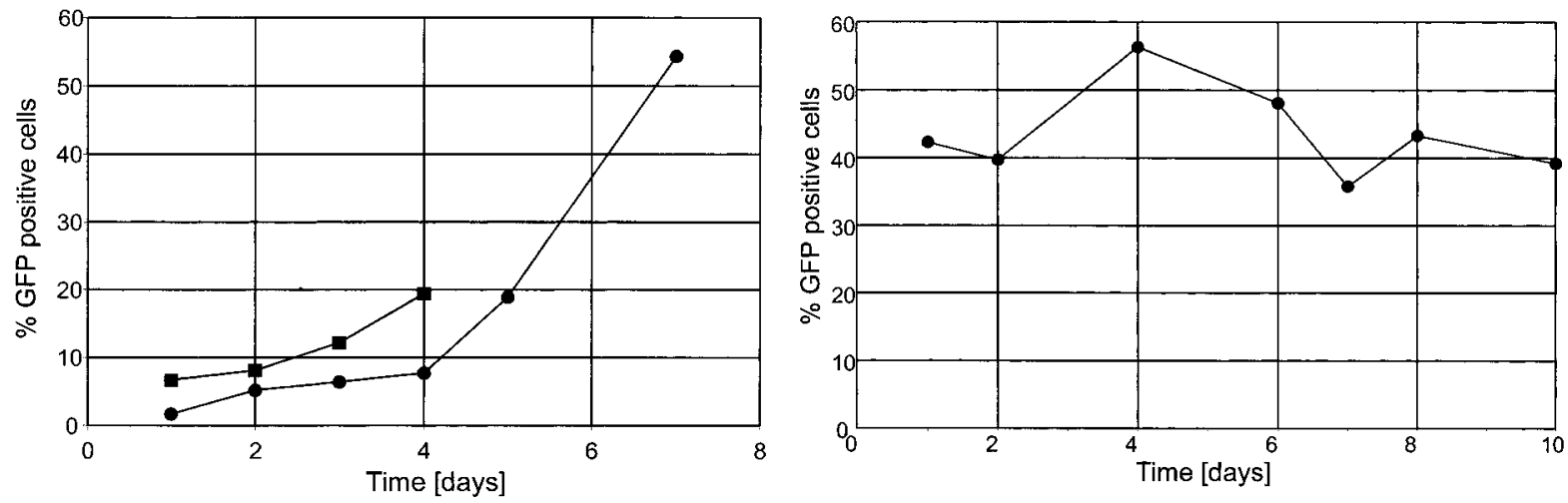

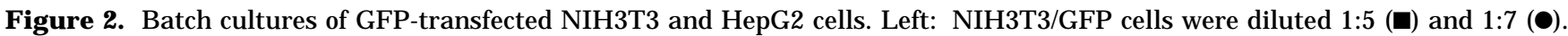
Right: HepG2/GFP cells were diluted 1:10. Culture supernatant was replaced on day 3 and 7.
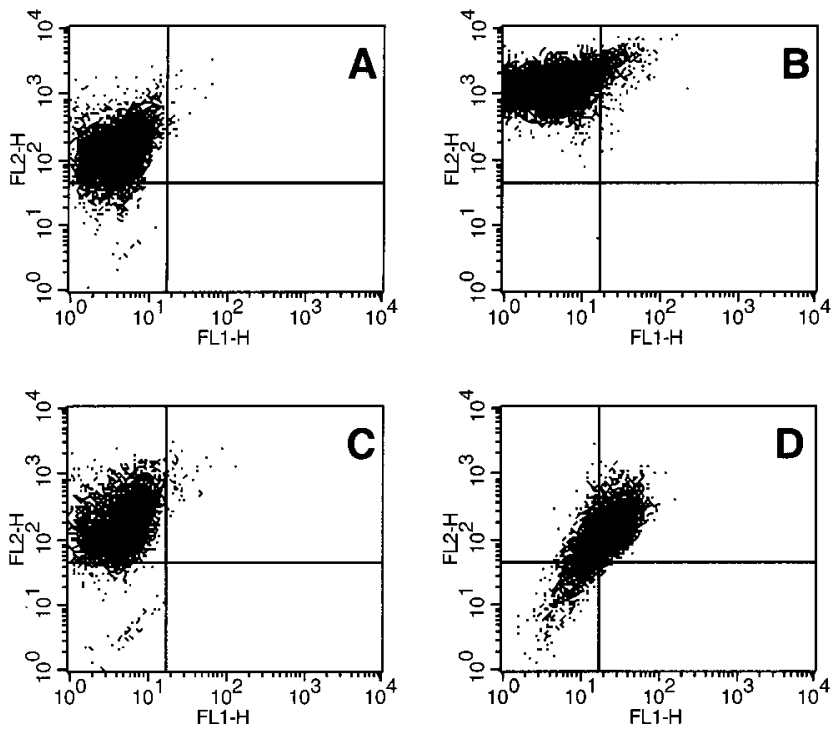

Figure 3. Two parameter measurements of GFP-transfected, PKH26-stained NIH3T3 cells: FI1, GFP fluorescence; FI2, PKH26 fluorescence. (A) Negative control stained with PKH26 after 3 days incubation. (B) NIH3T3/GFP cells directly after PKH26 staining. GFP positive cells are seen in the upper right quadrant. (C) NIH3T3/GFP cells after 3 days incubation: cells have divided and the GFP signal has disappeared. (D) NIH 3T 3/ GFP cells after 6 days of incubation: cells that have stopped dividing have increased GFP fluorescence, while those with higher division rates (in the lower left quadrant) have no GF P fluorescence.

to the results obtained with NIH3T3 cells, the fluorescent signal here seems to give a good indication of substrate supply: after each change of medium the fluorescence signal increases but remains around a fairly constant value. To verify the different production kinetics of these cell lines, we have stained both cell lines with PKH26, a fluorescent marker that is incorporated into the cell membrane. After each cell division the dye is distributed between daughter cells so that the fluorescent signal decreases. The resulting plots are shown in Figures 3 and 4: NIH3T3 cells have the highest GFP fluorescence in cells that have a high PKH26 signal, which means that they have undergone fewer divisions since staining, while HepG2 cells have comparable GFP fluorescence in cells with low and high PKH26 fluorescence.

These results demonstrate the importance of determining the production kinetics of the respective product in a cell line to be sorted. As a consequence of these findings, all measurements of GFP fluorescence in NIH 3 T3 cells were performed $24 \mathrm{~h}$ after confluence was reached. This hel ped to reduce the variation between consecutive
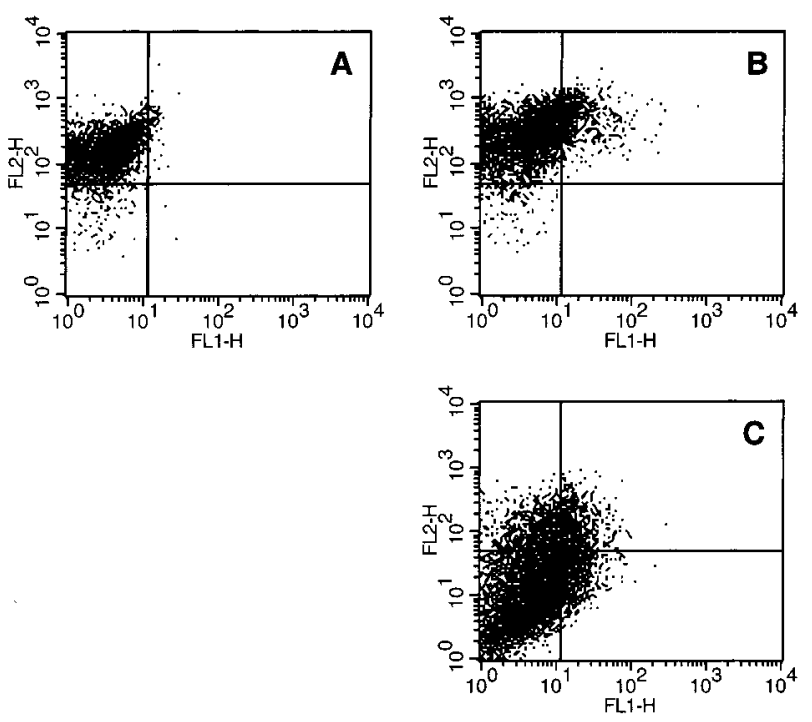

Figure 4. Two parameter measurements of GFP-transfected, PKH 26-stained HepG2 cells: FI1, GF P fluorescence; FI2, PKH26 fluorescence. (A) Negative control stained with PKH26. (B) HepG2/GFP cells stained with PHK26. (C) HepG2/GFP cells after 8 days incubation: the GFP positive cells are distributed equally over the whole $\mathrm{PKH} 26$ fluorescence range.

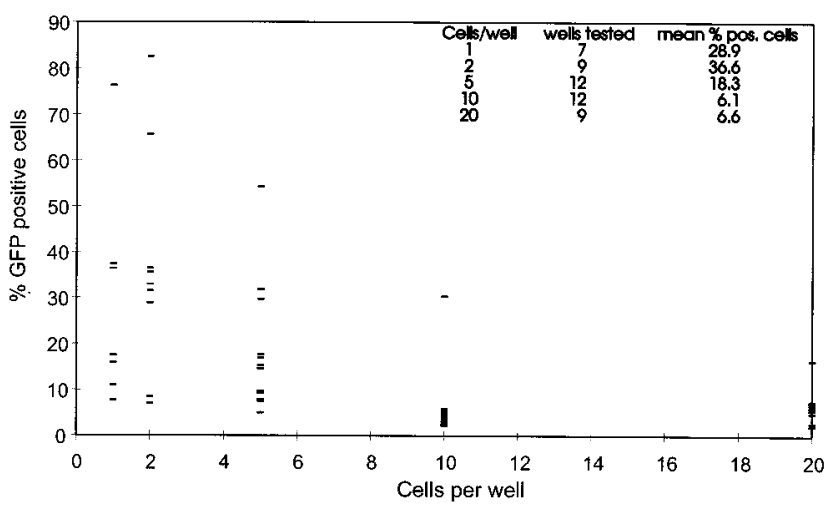

Figure 5. Cloning of NIH3T3/GFP cells. Cells were sorted directly into 96 -well plates at cell densities of $1,2,5,10$, and 20 cells/well. Cells were transferred into $2 \mathrm{~cm}^{2}$ wells and analyzed $24 \mathrm{~h}$ after confluence was reached.

measurements, although it could not be entirely eliminated for obvious reasons. In addition the increase in the percent of positive cells after confluence shows that more cells are positively transfected than can be measured due to the low expression rates.

Clone Sorting. As bulk sorting did not result in a stable, high-producing cell line, we sorted a pre-enriched 

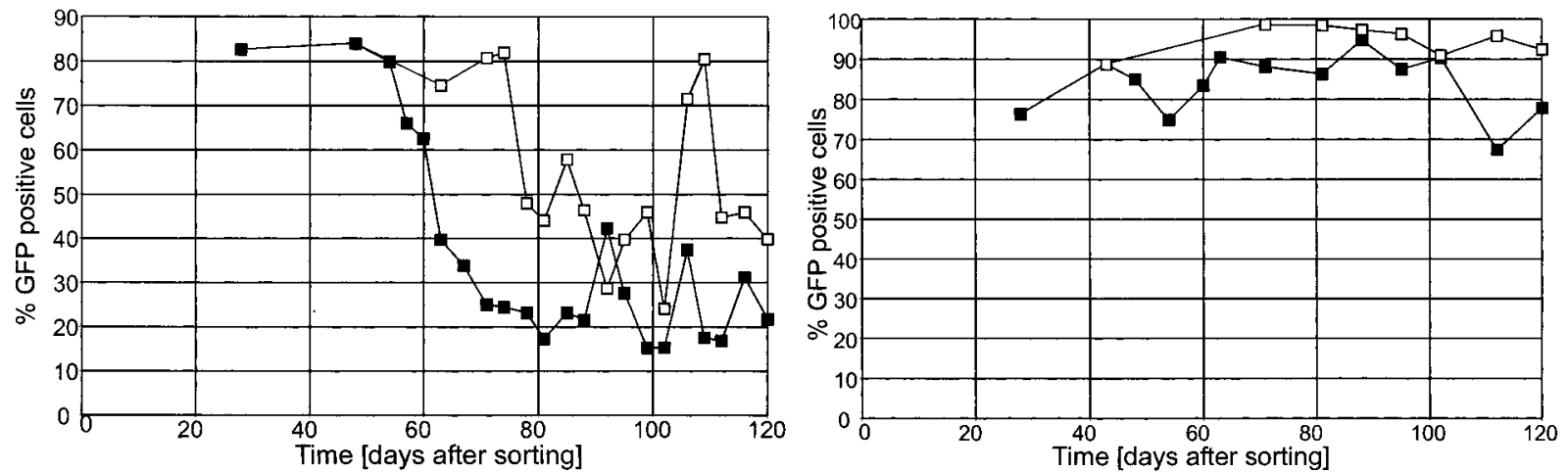

Figure 6. Percentage of GFP positive cells of cell lines NIH3T3/GFP/2B2 (left) and NIH3T3/GFP/3A2 (right). Cells were maintained in culture for 4 months ( $\square$ ) and subcloned by sorting once more $(\square)$.
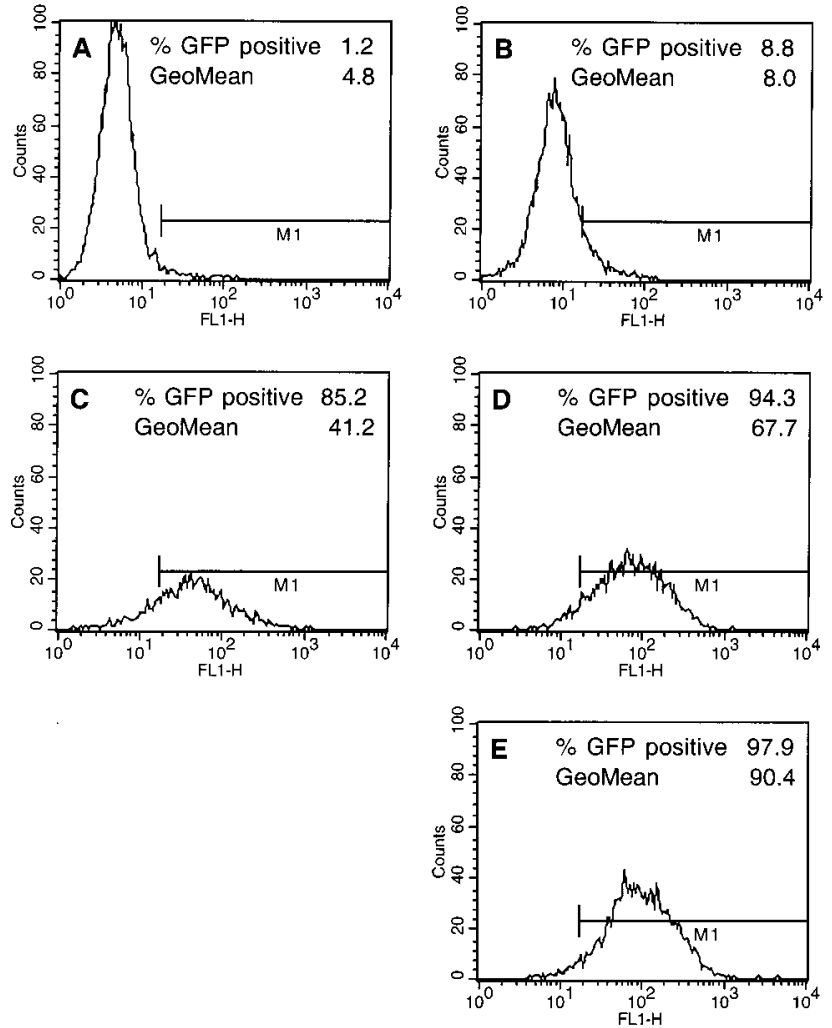

Figure 7. Flow cytometric analysis of GFP expression in NIH3T3 cells: A, NIH3T3 neg. control; B, NIH3T3/GFP after bulk sorting; C, subclone NIH3T3/GFP/2B2; D, subclone NIH3T3/ GFP/3A2; E, subclone NIH3T3/GFP/3A2/A.

population of cells that had been once bulk sorted, into 96-well plates so that $1,2,5,10$, and 20 cells ( 3 rows each) were sorted per well. The results are presented in Figure 5. As can be seen, the best clones were obtained when 1 or 2 cells were seeded per well. At higher cell numbers per well, it can be assumed that the resulting populations were not monoclonal, as the doning efficiency of this cell line was better than $20 \%$. The starting population had $5 \%$ positive cells, and most resulting populations coming from wells where 10 or 20 cells were sorted had comparable GFP expression. Only in wells with 1 or 2 cells, to a lower degree in wells with 5 cells, could high-producing clones be obtained. The two best clones, 2B2 and 3A2, were cultivated for 4 months and again subcloned by sorting to obtain even higher GFP expression. The results are shown in Figures 6 and 7. The number of GFP positive cells in clone 2B2 (Figure 6) started to decrease after 2 months and then stabilized between $20 \%$ and $30 \%$ GFP positive cells. This is a level still better than any obtained with bulk sorting. Repeated sorting resulted in a clone with comparable GFP fluorescence and prol onged stability. However, 20 days later this clone $2 \mathrm{~B} 2 / 2 \mathrm{~A} 6$ had al so lost $50 \%$ of its GF P positive cells.

The second clone 3A2 (Figure 6) had a stable expression of GFP and reached $80-90 \%$ GFP positive cells for 4 months. Repeated subcloning and sorting resulted in a clone 3A2/A that had a uniform, stable GFP expression with $95 \%$ GFP positive cells and an exceptionally high fluorescent signal (Figure 7E).

\section{Discussion}

Although GFP is frequently used for the optimization of protocols for transient gene expression, papers that deal with stable GFP expressing cells lines are comparatively rare. Lybarger et al. (1996) reported that no stable transfected NIH 3T3 cell line could be isolated when a plasmid was used that contained the GFP gene and a neomycin resistance marker, similar to the plasmid that we used. Only when they constructed a GFP-hygromycin fusion protein could they isolate a stable transfectant. However, for most recombinant products, such a fusion protein is not desirable. It has been described in several papers (f. i. Gu et al., 1992; Kromenaker and Srienc, 1994) that high-producing cell lines tend to have lower growth rates. This alone can explain the instability of GFP expression even under selective pressure. Cells that somehow manage to express the marker gene without expressing or while expressing low amounts of GFP will have a higher growth rate and thus overgrow producers. The conclusion from these papers and our data is that, to select stable high producers, single cells have to be isolated. Single cloned cells may still be unstable, but if a mixed population is present, the low producers will outgrow the high producers within 1 or 2 months (Kromenaker and Srienc, 1994).

The main problem of GFP analysis and sorting is the low fluorescence signal intensity. This was described before by Subramanian and Srienc (1996). It can be deduced from our results that many cells, although positively transfected, have a signal intensity too low to discriminate from autofluorescence. This is proven by the fact that the number of GFP positive cells increases when cells become confluent and reduce their growth rate and decreases again when cells are re-inoculated. We conclude that it is important to characterize the production kinetics of a desired product in a given cell line to select the optimal time point for analysis and sorting. On the other hand the knowledge about the given kinetics might be exploited to change the kinetics if a different production pattern is required. In our case we might have 
decided that it would be desirable to have a cell line with a high production rate at high growth rates. In this case we should have sorted when cells were in exponential growth.

\section{Conclusions}

To isolate high-producing cell lines with a fluorescenceactivated cell sorter, several points need to be considered:

For long time stability of cell lines, it is important to sort and clone single cells. Otherwise non- or low producers will outgrow high producers. However, if positive cells are very rare or unstable, it may be beneficial to enrich by bulk sorting before cloning. For short time experiments it will be sufficient to sort a large number of cells in bulk.

The kinetics of product formation of a given product in a given cell line should be characterized in order to identify the optimal time point for analysis and sorting. This knowledge might also be used to try to change the production kinetics, if desired.

Exclusion of doublets and aggregates ( $)$ antscheff et al., 1993) by using a gate set on the Forward Scatter Width signal obtained by pulse processing helps to isolate only positive cells.

\section{References and Notes}

Cherlet, M.; Kromenaker, S. J .; Srienc, F. Surface IgG content of murine hybridomas: Direct evidence for variations of antibody secretion rates during the cell cycle. Biotechnol. Bioeng. 1995, 47, 535-540.

Gu, M. B.; Kern, J . A.; Todd, P.; Kompala, D. S. Effect of amplification of dhfr and lacZ genes on growth and $\beta$-galactosidase expression in suspension cultures of recombinant CHO cells. Cytotechnology 1992, 9, 237-245.

Gu, M. B.; Todd, P.; Kompala, D. S. Foreign gene expression during the cell cycle phases in recombinant $\mathrm{CHO}$ cells. Biotechnol. Bioeng. 1993, 42, 1113-1123.

Gubin, A. N.; Reddy, B.; Njoroge, J . M.; Miller, J . L. Long-term, stable expression of green fluorescent protein in mammalian cells. Biochem. Biophys. Res. Commun. 1997, 236, 347-350.

J antscheff, P.; Winkler, L.; Karawajew, L.; Kaiser, G.; Böttger, V.; Micheel, B. Hybrid hybridomas producing bispecific antibodies to CEA and peroxidase isolated by a combination of HAT medium selection and fluorescence activated cell sorting. J . Immunol. Methods 1993, 163, 91-97.

Kim, S. J .; Kim, N. S.; Ryu, C. J .; Hong, H. J .; Lee, G. M. Characterization of chimeric antibody producing $\mathrm{CHO}$ cells in the course of dihydrofolate reductase-mediated gene amplification and their stability in the absence of selective pressure. Biotechnol. Bioeng. 1998, 58, 73-84.

Kromenaker, S. J .; Srienc, F. Stability of producer hybridoma cell lines after cell sorting: a case study. Biotechnol. Prog. 1994, 10, 299-307.

Lybarger, L.; Dempsey, D.; Franek, K. J .; Chervenak, R. Rapid generation and flow cytometric analysis of stable GFPexpressing cells. Cytometry 1996, 25, 211-220.

Marder, P.; Maciak, R. S.; Fouts, R. L.; Baker, R. S.; Starling, J.J. Selective cloning of hybridoma cells for enhanced immunogl obulin production using flow cytometric cell sorting and automated laser nephel ometry. Cytometry 1990, 11, 498505.

McKinney, K. L.; Dilwith, R.; Belfort, G. Manipulation of heterogeneous hybridoma cultures for overproduction of monoclonal antibodies. Biotechnol. Prog. 1991, 7, 445-454.

Morrison, C. J .; McMaster, W. R.; Piret, J. M. Differential stability of proteolytically active and inactive recombinant metalloproteinase in Chinese Hamster Ovary cells. Biotechnol. Bioeng. 1997, 53, 6, 594-600.

Rosenblatt, J . I.; Hokanson, J . A.; McLaughlin, S. R.; Leary, J . $F$. Theoretical basis for sampling statistics useful for detecting and isolating rare cells using flow cytometry and cell sorting. Cytometry 1997, 27, 233-238.

Sen, S.; Hu, W.-S.; Srienc, F. Flow Cytometric study of hybridoma cell culture: Correlation between cell surface fluorescence and IgG production rate. Enzyme Microb. Technol. 1990, 12, 571-576.

Subramanian, S.; Srienc, F. Quantitative analysis of transient gene expression in mammalian cells using the green fluorescent protein. J . Biotechnol. 1996, 49, 137-151.

Zhang, G.; Gurtu, V.; Kain, S. R. An enhanced fluorescent protein allows sensitive detection of gene transfer in mammalian cells. Biochem. Biophys. Res. Commun. 1996, 227, 707-711.

Accepted J une 28, 1999.

BP990089G 